\title{
Effects of hyperthermic baths on depression, sleep and heart rate variability in patients with depressive disorder: a randomized clinical pilot trial
}

\author{
Johannes Naumann ${ }^{1 *}$, Julian Grebe ${ }^{2}$, Sonja Kaifel ${ }^{2}$, Tomas Weinert ${ }^{2}$, Catharina Sadaghiani ${ }^{1}$ (I) and Roman Huber ${ }^{2}$
}

\begin{abstract}
Background: Despite advances in the treatment of depression, one-third of depressed patients fail to respond to conventional antidepressant medication. There is a need for more effective treatments with fewer side effects. The primary aim of this study was to determine whether hyperthermic baths reduce depressive symptoms in adults with depressive disorder.
\end{abstract}

Methods: Randomized, two-arm placebo-controlled, 8-week pilot trial. Medically stable outpatients with confirmed depressive disorder (ICD-10: F32/F33) who were moderately depressed as determined by the 17-item Hamilton Scale for Depression (HAM-D) score $\geq 18$ were randomly assigned to 2 hyperthermic baths $\left(40^{\circ} \mathrm{C}\right)$ per week for 4 weeks or a sham intervention with green light and follow-up after 4 weeks. Main outcome measure was the change in HAM-D $D_{\text {total score }}$ from baseline (T0) to the 2-week time point (T1).

Results: A total of 36 patients were randomized (hyperthermic baths, $n=17$; sham condition, $n=19$ ). The intentionto-treat analysis showed a significant $(P=.037)$ difference in the change in HAM-D total score with 3.14 points after 4 interventions (T1) in favour of the hyperthermic bath group compared to the placebo group.

Conclusions: This pilot study suggests that hyperthermic baths do have generalized efficacy in depressed patients.

Trial registration: DRKS00004803at drks-neu.uniklinik-freiburg.de, German Clinical Trials Register (registration date 2016-02-02), retrospectively registered.

Keywords: Depression, Hyperthermic baths, Heart rate variability, RCT

\section{Background}

Depression is the leading cause of disability worldwide, and a major contributor to the global burden of disease, with more than 350 million people of all ages suffering from depression [1]. The associated economic burden is estimated at $\$ 317$ billion annually [2], and antidepressant medications have become the most commonly prescribed treatment in medical practice [3]. In addition, psychiatric conditions, particularly depressive disorders,

\footnotetext{
* Correspondence: johannes.naumann@uniklinik-freiburg.de

${ }^{1}$ Interdisciplinary Center for Treatment and Research in Balneology, Institute for Environmental Health Sciences and Hospital Infection Control, Medical Faculty, Medical Center University of Freiburg, Breisacher Straße 115b, Freiburg im Breisgau 79106, Germany

Full list of author information is available at the end of the article
}

are associated with increased prevalence of chronic diseases and often precipitate or exacerbate these conditions [4]. Depression is a known risk factor for the development of cardiovascular disease (CVD) [5], and patients with CVD and depression have a two to threefold increased risk of future cardiac events compared to cardiac patients without depression [6].

Alterations in the autonomic nervous system have been hypothesized to be an underlying physiological mechanism that may partly explain these unfavorable health outcomes among depressed persons. Such alterations are believed to disturb circadian functioning, sleep and temperature physiology [7]. Depressed persons are found to have a reduced heart rate variability (HRV), a well-known prognostic risk factor for CVD and 
mortality; however, it is unclear whether this is a consequence of the disorder or due to antidepressant medication [8, 9]. Most depressed patients also report disturbances in their sleep, such as difficulties in falling asleep, waking during the night or early morning awakenings. Thus, sleep disturbance is an important mechanism contributing to depression [7, 10]. Furthermore, body core temperature in depressed patients is elevated during the night; thus, change of body temperature might influence depression $[11,12]$. Despite advances in the treatment of depression, one-third of depressed patients fail to respond to conventional antidepressant medication [13].

Moreover, current treatments come at the cost of significant central nervous side effects, further highlighting the need for more effective treatments with fewer side effects [14].

As a conclusion, there is a need for additional (nonpharmacological) treatment of depressive patients that positively influence cardiovascular risk factors, HRV and quality of sleep. Evidence suggests that hyperthermic baths (HTB) and other forms of whole body hyperthermia (WBH) have antidepressant effects, mediated through changes in circadian functioning and temperature physiology, although the underlying mechanisms remain unclear $[15,16]$.

Results of a non-controlled HTB study with 20 depressive patients showed an improvement in the 21-item Hamilton Depression Scale [17] after five baths [18]. Furthermore, HTB (especially before bedtime) improved sleep in healthy subjects [19-22], insomniac people [23, 24] and elderly patients with vascular dementia [25]. In a further non-controlled study using a radiant system to induce WBH, a single session showed a significant reduction in the Centers for Epidemiologic Studies Depression Scale (CES-D) [26] in 16 depressive patients [27].

Results of these uncontrolled studies are promising; therefore, we conducted this randomized sham-placebocontrolled pilot study that included assessment of depressive symptoms, sleep quality and HRV parameters. Our hypotheses were 2-fold. First, we expected HTB to lower depressive symptoms. Second, we expected this to be mediated by improved sleep and circadian functioning, evoked by changes in HRV parameters.

\section{Methods}

\section{Study design and participants}

Because of the unknown effect size and in order to evaluate the feasibility of recruitment and assessment procedures a pilot study was conducted.

This pilot study was a single-site, parallel-group, randomized controlled trial of HTB vs sham-placebo (green light) for patients with a confirmed diagnosis of depression according to ICD-10 (F32/F33) of at least 4 weeks duration. Recruitment via public announcements took place from June 2013 until September 2013.

Eligible participants were men and women between the ages of 18 and 65 who had been on a consistent antidepressant regimen or had been off antidepressant therapy for at least 4 weeks prior to baseline. No changes in antidepressant treatment were allowed during the study. All participants were required to have a total score $\geq 18$ and a score $\geq 2$ on item 1 (Depressed Mood) at screening and at baseline, assessed by the 17-item Hamilton Scale for Depression (HAM-D). Exclusion criteria included the presence of severe concomitant disease, epileptic disorders, organic psychotic disorders, schizophrenia, hallucinations, bipolar disorders, dissociative personality disorder, suicidal thoughts, abuse of alcohol or other drugs within the last 6 months, use of $ß$-blockers or corticosteroids, open wounds, heat urticaria, pregnancy, lactation, aversion to hot baths and participation in clinical trials in the 8 weeks preceding the study.

\section{Ethics statement}

Ethical approval was obtained from the local Ethics Committee (Ethics-Commission Medical Center University of Freiburg; 96/13; 22.04.2013). The study was retrospectively registered in the German Clinical Trials Register (DRKS) with the registration number DRKS00004803. The study was conducted in accordance with the Declaration of Helsinki and local laws and regulations. All participants gave written informed consent. The full study protocol can be found in Additional file 1 .

\section{Interventions}

Patients were randomly assigned to receive either HTB or green light therapy (sham condition) for 4 weeks with 2 interventions per week (8 interventions in each group). Follow-up took place 4 weeks after the last intervention (see Table 1). Patients were told that two promising treatments are compared in the study.

\section{Hyperthermic baths}

The hyperthermic baths were applied as head-out-ofwater-immersion in a $40{ }^{\circ} \mathrm{C}$ pool at a spa center near Freiburg, Germany. All the baths were conducted in the afternoon (14:00-18:00). Five patients were able to sit in the pool at a time. The baths were taken until the participants noticed discomfort, the target being $30 \mathrm{~min}$. Directly after the bath, the participants were accompanied to a nearby resting room, where they lay down on a resting lounger and were wrapped in warm blankets with 2 conventional $0.7 \mathrm{l}$ hot water bottles (abdomen, thighs) filled with boiling water for at least another $30 \mathrm{~min}$ to keep the body temperature elevated. After 20-30 min in a hyperthermic bath with a water temperature of $40{ }^{\circ} \mathrm{C}$ a 
Table 1 Diagram of the study protocol

\begin{tabular}{|c|c|c|c|c|c|}
\hline & & & 2 weeks & 2 weeks & 4 weeks \\
\hline & Screening & Baseline/Randomization & After 4 interventions & & Follow-up \\
\hline & & T0 & T1 & & T2 \\
\hline HTB target $(n)^{a}$ & & & 4 & 4 & \\
\hline 17-item HAM-D & $x$ & $x$ & $x$ & & $x$ \\
\hline Medical history ${ }^{b}$ & & $x$ & & & \\
\hline HRV & & $x$ & $x$ & & $x$ \\
\hline Global judgement of efficacy & & & $x$ & & $x$ \\
\hline Global judgement of tolerability & & & $x$ & & $x$ \\
\hline Adverse events $^{d}$ & & & $x$ & $x$ & $x$ \\
\hline
\end{tabular}

Assessments of psychometry and heart rate variability parameters (HRV) were conducted at baseline (T0), after 2 weeks of treatment (T1), and 4 weeks after discontinuation of treatment (T2). Note that assessments were not conducted after 4 weeks of treatment

${ }^{a}$ HTB: hyperthermic baths; temperature of bath; ear-temperature before/after bath; ear-temperature after rest; duration of bath (target 30 min); duration of rest

${ }^{b}$ Duration of depression; approved diagnosis family/consulting physician; intake of psychometric drugs no/yes; psychotherapy no/yes; psychiatric hospital stays

' Application of portable ECG for overnight measurement

${ }^{\mathrm{d}}$ Adverse events were documented before and after each treatment

raise in core body temperature of $1.7^{\circ} \mathrm{C}$ can be expected [28].

The following parameters were controlled and documented: water temperature in the bath, duration of bath and resting time.

\section{Core body temperature}

Core body temperature was measured with an infraredear-thermometer (Thermoscan ${ }^{\circ}$, Type: 6021, Braun $\mathrm{GmbH})$, before and after the bath and after resting.

\section{Sham intervention}

Participants received a sham green LED light intervention ( $<400 \mathrm{Lux} ;<40 \mathrm{~min})$ in group settings in a sitting position at a university outpatient's department.

Therapeutic effects can be expected for therapies at 10 000 Lux/30 min per day [29].

\section{Outcome measures}

Unblinded assessments were performed at the following 3 time points (see Table 1): before the start of HTB treatment (T0), immediately on completion of the 2week treatment interval (T1), according to results that effects are supposed to appear early, [15] and 4 weeks after the end of treatment (T2).

The primary outcome was determined to be the change in HAM- $\mathrm{D}_{\text {total score }}$ at T1 relative to T0. Secondary efficacy outcome measures were (1) change in HAM- $\mathrm{D}_{\text {total }}$ score at $\mathrm{T} 2$ relative to $\mathrm{T} 0$ to investigate whether immediate responses would last after treatment discontinuation, (2) change in HAM-D subscales (T1 and T2): Insomnia as a symptom was measured by a HAM-D subscale (range of scores $0-4$ ) defined by the cluster of items 4 (Early in the Night), 5 (Middle of the Night), and 6 (Early Hours of the Morning). Low mood, a core symptom of depression, was assessed by a HAM-
D subscale consisting of items 1 (Depressed Mood), 2 (Guilt), 3 (Suicide), 7 (Loss of Interest), and 10 (Anxiety). Somatic complaints were measured by a HAM-D subscale defined by the cluster of items 11 (Anxiety Somatic), 12 (Gastro-intestinal Symptoms), and 13 (General Symptoms) [30, 31].

\section{Heart rate variability outcome measures}

Further secondary endpoints were standard HRV parameters, notably total heart rate variability (SDNN; standard deviation of normal-to-normal R-R intervals), low frequency (LF), high frequency (HF), LF/HF ratio and heart rate (HR; mBPM) during sleep (T1 and T2: 21:0004:00, 21:00-0:30 and 0:30-4:00). We chose these nighttime intervals because we were interested in sleep patterns and because these measurements are supposed to show less artefacts and influence of daily life activity.

HRV was measured with a portable high-frequency recorder "Medilog ${ }^{\bullet}$ AR12plus" (Schiller Medizintechnik $\mathrm{GmbH}$, Germany), with a resolution of $1000 \mathrm{~Hz}$. Electrodes were fixed at 5 locations (upper and lower end of sternum, below lateral third of left clavicle, under ribs right and left of left mamillar line). HRV analysis was performed with the program "Medilog Darwin Version 1.13.4".

\section{Global judgement of efficacy and tolerability}

After 4 treatments (T1) and 4 weeks after end of treatment (T2), participants were asked to rate the efficacy and tolerability of the intervention on a 5-point scale (1 indicates very good; 2 , good; 3 , moderate; 4 , absent; 5 , worsening).

\section{Adverse events}

Adverse events (AE) were documented before and after each treatment by two unblinded assistants. 


\section{Statistical analyses \\ Sample size}

We calculated the sample size assuming an effect of one standard deviation (SD) between the groups based on a two-sided $t$ test with an $\alpha$-risk level of .05 and a statistical power of $80 \%$, requiring 17 participants per group. With an expected dropout rate of $20 \%, 22$ participants per group were chosen. Because of slow recruitment and logistical difficulties, however, the study was halted before the target sample size was attained.

\section{Randomization and blinding}

Randomization codes were computer-generated by an independent biometric center. Allocation was performed with opaque sealed envelopes that were randomly chosen by the participants. Both therapies could not be blinded. Data management and analyses were performed blinded to treatment allocation.

\section{Data analyses}

Efficacy parameters were analyzed based on the intention-to-treat (ITT) population, defined as all allocated participants, applying the last-observation-carriedforward approach to impute missing data. Baseline characteristics were compared using 2 -sided $t$ tests for continuous data and $X^{2}$ statistics. The per-protocol (PP) population was defined as all participants who had a complete dataset for the relevant parameters and had participated in at least $75 \%$ of the treatments, meaning at least 3 of 4 treatments for T1, and at least 6 of 8 treatments for T2.

Based on the fact that the HTB group was expected to show better performance than the placebo group, we justified 1-sided testing on the primary and secondary outcomes of depression ratings. We report $P$-values with the significance level set at $P<.05$ and Cohen $d$ [32] as effect size. Correlation analyses were used to explore associations between HAM-D scores and HRV parameters. Secondary analyses were not adjusted for multiple comparisons and should therefore be regarded as descriptive and exploratory. Where not otherwise indicated, data are expressed as mean (SD). Statistical analyses were performed using SPSS ${ }^{\oplus}$, Version 22, for WindowsTM.

\section{Results}

\section{Study population}

We included and randomized 36 depressed patients (HTB, $n=17$; placebo, $n=19$ ). Six patients (HTB, $n=3$; placebo, $n=3$ ) discontinued treatment before T1; none were lost to follow-up (see Fig. 1). Randomization was balanced with respect to demographic and clinical characteristics (Table 2).
Treatment effect on core body temperature

Core body temperature rose from $36.6{ }^{\circ} \mathrm{C}$ before the baths to $39.1{ }^{\circ} \mathrm{C}$ directly after the baths (mean change $2.43[0.4]$ ) and maintained at $37.7^{\circ} \mathrm{C}$ (mean change 1.06 [0.5]) after rest. The mean temperature of the bath was $40.2{ }^{\circ} \mathrm{C}(0.3)$. Mean duration of baths was 22.6 (3.5) $\mathrm{min}$ and resting time amounted to 33.2 (6.3) $\mathrm{min}$.

\section{Primary outcome}

The ITT analysis showed a significant $(P=.037)$ difference in the change in HAM-D $D_{\text {total score }}$ with 3.14 points after 4 interventions in favour of the HTB group compared to the placebo group (see Fig. 2). This result was confirmed by the PP analysis $(P=.031)$ and the subscales HAM-D $D_{\text {insomnia }}(P=.048)$ and HAM-D $D_{\text {mood }}(P=.045)$ showed a significant improvement compared to the placebo group (Additional file 2: Table S1). Posthoc subgroup-analyses according to HAM-D scores quartiles (median) revealed the greatest treatment effect in quartiles 3 and 4. We found a difference of 7.88 (7.5) for HAM-D $>22$ at baseline in the HTB group $(n=8)$ and of 1.71 (1.7) in the placebo group $(n=7)(d=1.10 ; 95 \% \mathrm{CI}$, 0.02 to 2.19) and a difference of 12.5 (8.2) for HAM-D $>26$ at baseline in the HTB group $(n=4)$ and of 1.66 (2.3) in the placebo group $(n=3) ;(d=1.67 ; 95 \% \mathrm{CI}$, -0.07 to 3.40$)$.

\section{Secondary outcomes}

The positive effect of HTB treatment on depression remained stable until 4 weeks after the intervention without, however, reaching statistical significance (Table 3). For results of the PP analysis, see Additional file 2: Table S2 and Figures S1, S2.

\section{Heart rate variability}

Throughout the study, the data analysis (PP) did not reveal significant differences in HRV parameters between the HTB and the placebo group. Interestingly, the improvement of sleep quality (HAM-D $\mathrm{D}_{\text {insomnia }}$ ) within 3 days of HTB treatment significantly correlated with an increase of nocturnal pulse rate (pearson -.63; $\left.P_{2 \text {-tailed }}=.020\right)$ and overnight drop of the parameters LF (pearson $.65 ; P_{2 \text {-tailed }}=.016$ ) and total variability of HRV (SDNN; pearson .62; $\left.P_{2 \text {-tailed }}=.024\right)$. There was no correlation of these parameters in the sham condition.

\section{Global judgement of efficacy and tolerability}

The global judgement by the participants showed no significant difference between the groups, with good to moderate efficacy (HTB 2.6 (0.9); placebo 2.8 (1.0); $P_{2 \text { - }}$ tailed $=.62$ ) and tolerability (HTB 2.2 (1.1); placebo 1.6 $\left.(0.9) ; P_{2 \text {-tailed }}=.17\right)$ after 4 interventions and good to moderate efficacy 4 weeks after intervention (HTB 2.8 (1.15); placebo $\left.2.7(1.10) ; P_{2 \text {-tailed }}=.84\right)$. 


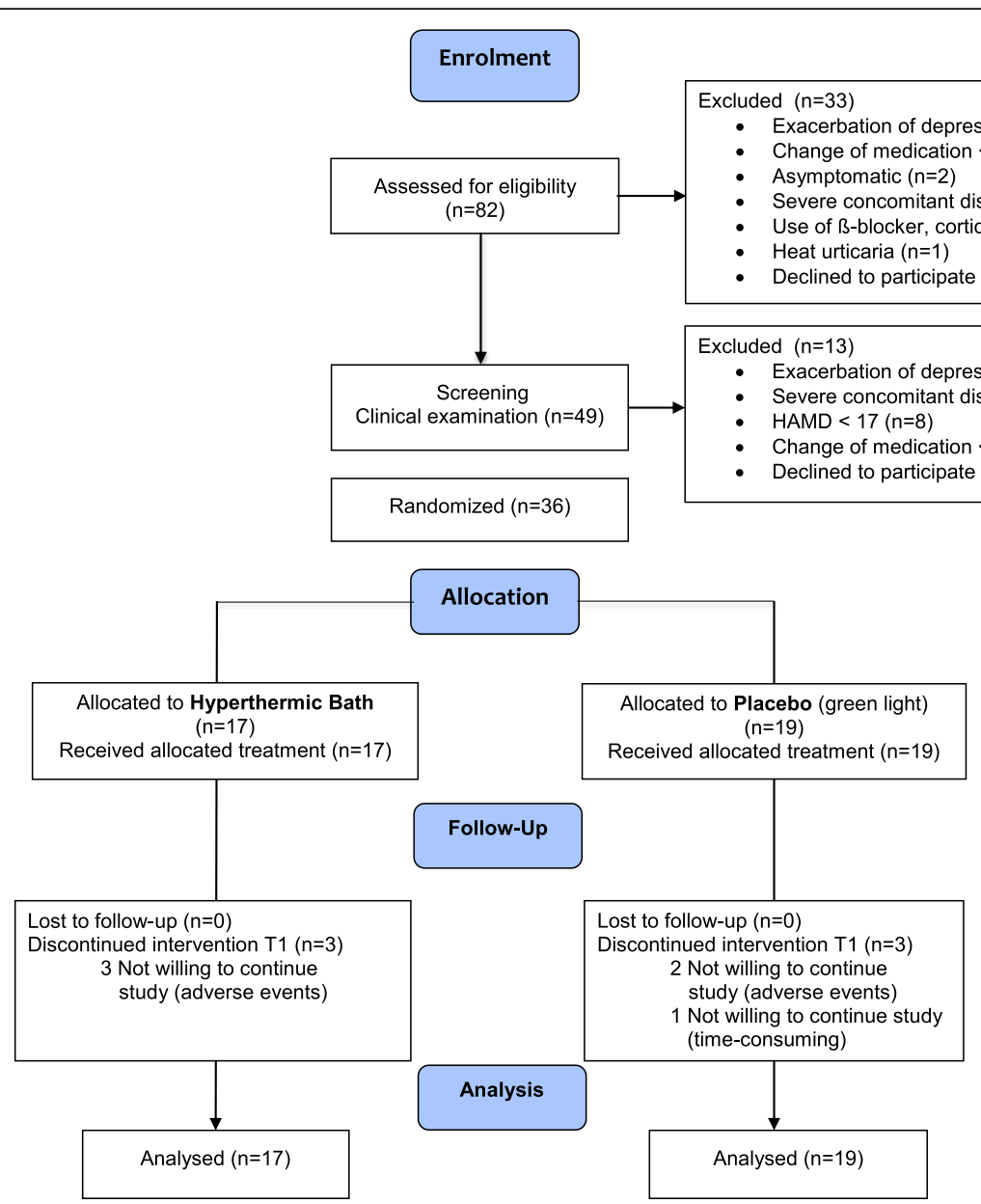

Fig. 1 CONSORT Flow Diagram of study participants

Table 2 Baseline demographic and clinical characteristics of the 36 randomized study patients (intention-to-treat population) by treatment assignment

\begin{tabular}{|c|c|c|c|c|}
\hline & $\begin{array}{l}\text { Overall } \\
N=36\end{array}$ & $\begin{array}{l}\text { HTB Group } \\
(N=17)\end{array}$ & $\begin{array}{l}\text { Placebo Group } \\
(N=19)\end{array}$ & $P$ Value $^{a}$ \\
\hline Gender (female/male), n & $28 / 8$ & $14 / 3$ & $14 / 5$ & 0.70 \\
\hline Age (years) & $47(11.9)$ & $44(13.0)$ & 49 (10.6) & 0.22 \\
\hline BMI $\left(\mathrm{kg} / \mathrm{m}^{2}\right)$ & $24(5.2)$ & $24(3.9)$ & $25(6.3)$ & 0.72 \\
\hline Duration of depression (months) & $93(85.7)$ & $98(92.5)$ & $89(82.7)$ & 0.79 \\
\hline Use of psychopharmaca (\%) & 64 & 65 & 63 & 1 \\
\hline HAM-D total score & $22.36(3.7)$ & $23.06(3.9)$ & $21.74(3.4)$ & 0.29 \\
\hline HAM-D insomnia & $3.36(2.0)$ & $3.35(1.8)$ & $3.37(2.3)$ & 0.98 \\
\hline HAM-D mood & $10.47(2.1)$ & $10.82(1.8)$ & $10.16(2.3)$ & 0.35 \\
\hline HAM- $D_{\text {somatic }}$ & $3.92(1.2)$ & $4.00(1.3)$ & $3.84(1.2)$ & 0.71 \\
\hline Heart rate $(21: 00-04: 00)^{b}$ & $72.77(10.8)$ & $73.20(9.4)$ & 72.39 (12.2) & 0.84 \\
\hline HRV LF (21:00-04:00) $)^{\mathrm{b}}$ & 490.30 (329.8) & 485.70 (353.6) & 494.33 (319.3) & 0.94 \\
\hline HRV HF (21:00-04:00) & $161.94(133.8)$ & $156.47(154.6)$ & $166.72(117.6)$ & 0.84 \\
\hline HRV SDNN (21:00-04:00) & $55.14(16.5)$ & $55.33(15.9)$ & $54.97(17.6)$ & 0.95 \\
\hline
\end{tabular}

Where not otherwise indicated, data are shown as mean and standard deviation (SD)

Abbreviations:

${ }^{a}$ Calculated as comparisons of HTB and placebo, using 2 -tailed $t$ tests (continuous variables) or $x^{2}$ tests (discrete variables)

${ }^{\mathrm{b}}$ Night-time intervals 


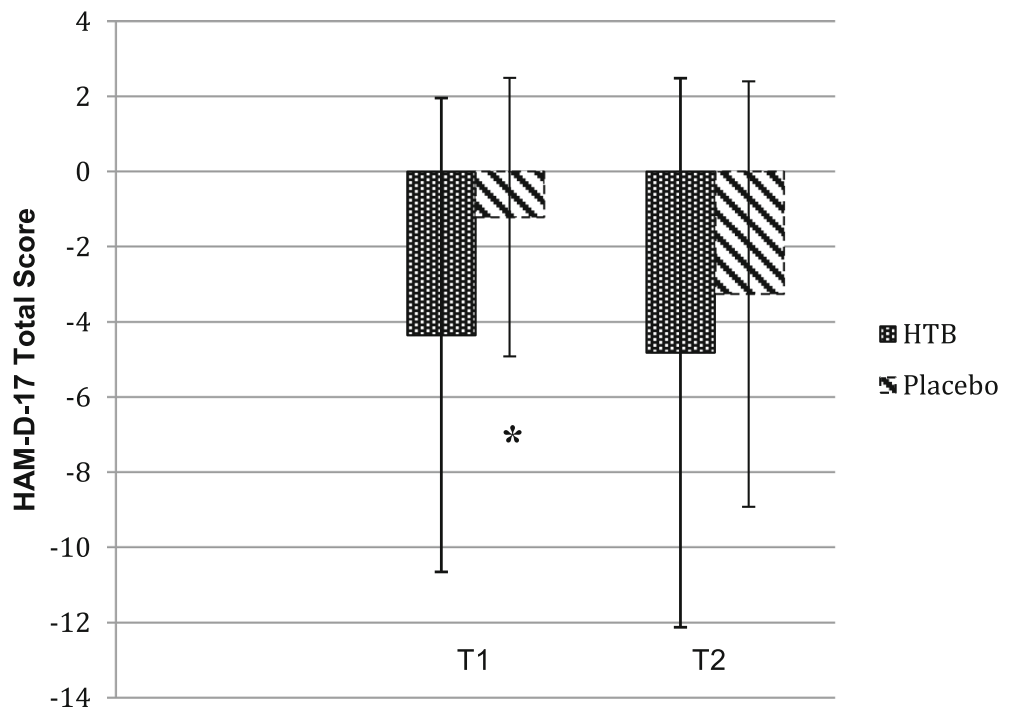

Fig. 2 Change scores in the 17-Item Hamilton Scale for Depression (HAM-D-17) from baseline (T0) in the hyperthermic bath group compared to the placebo group (intention-to-treat population; ${ }^{a} \mathrm{~N}=36$ ). T1 indicates after 2 weeks of treatment (4 hyperthermic baths (HTB) vs sham intervention with green light (placebo); T2, 4 weeks after discontinuation of treatment (follow-up). ${ }^{\text {TT }}$ The intention-to-treat analysis used last-observation-carried-forward.Error bars indicate standard deviation.* $P=0.037$

\section{Adverse events}

AE were reported by 21 participants, of which 12 (86\%) were assigned to the HTB group and 9 (56\%) to the placebo group (Additional file 2: Table S3). No serious AE were reported by either group. There was no significant difference between the groups $\left(P_{2 \text {-tailed }}=118\right)$. Typical $\mathrm{AE}$ in the HTB group were discomfort during the baths such as dizziness, tachycardia, tingling in the extremities and strong perspiration and thus mainly attributable to the cardiovascular system. Additionally, patients reported minor headache and nausea or a feeling of oppression, poorer sleep and increased sweating on the following days. Typical AE in the sham condition were afterimages lasting a few seconds to minutes after application, head pressure (headache) and worse sleep quality during the following night. $\mathrm{AE}$ resulted in 3 dropouts (DO) in the HTB group ( $\mathrm{DO}_{1}$ weight loss, headache, exertion the day after treatment; $\mathrm{DO}_{2}$ anxiety at night; $\mathrm{DO}_{3}$ headache) and in 2 dropouts in the placebo group $\left(\mathrm{DO}_{1}\right.$ feeling of tension; $\mathrm{DO}_{2}$ feeling of aggression). One dropout in the placebo group was due to lack of time. Compliance was good with a medium number of treatments of 7.3 (1.4) in the HTB group and of $7.8(0.5)$ in the placebo group.

\section{Discussion}

The main result of this pilot study was a moderate but significant improvement of 3.1 points in HAM-

Table 3 17-Item Hamilton Scale for Depression: Differences between baseline and T1 (after 4 interventions) and T2 (4 weeks after treatment) in the hyperthermic bath group compared to the placebo group (intention-to-treat population; ${ }^{\mathrm{a}} \mathrm{N}=36$ )

\begin{tabular}{|c|c|c|c|c|c|c|c|c|}
\hline & \multicolumn{3}{|c|}{$\mathrm{T} 1$} & \multicolumn{5}{|c|}{$\mathrm{T} 2$} \\
\hline & $\begin{array}{l}\text { HTB Group } \\
(n=17)\end{array}$ & $\begin{array}{l}\text { Placebo Group } \\
(n=19)\end{array}$ & $p^{\S}$ & $\begin{array}{l}\text { Cohen } \\
d^{c}\end{array}$ & $\begin{array}{l}\text { HTB Group } \\
(n=17)\end{array}$ & $\begin{array}{l}\text { Placebo Group } \\
(n=19)\end{array}$ & $p^{\S}$ & $\begin{array}{l}\text { Cohen } \\
d^{c}\end{array}$ \\
\hline${ }^{1} \mathrm{HAM}-\mathrm{D}_{\text {total score }}^{\mathrm{b}}$ & $4.35(6.3)$ & $1.21(3.7)$ & $0.037^{*}$ & $0.62[-0.05-1.29]$ & $4.82(7.3)$ & $3.26(5.7)$ & 0.238 & $0.24[-0.42-0.90]$ \\
\hline${ }^{2} H A M-D_{\text {insomnia }}^{b}$ & $0.94(1.8)$ & $0.16(1.0)$ & 0.053 & $0.55[-0.11-1.22]$ & $0.88(1.7)$ & $0.58(1.8)$ & 0.306 & $0.17[-0.49-0.83]$ \\
\hline${ }^{2} \mathrm{HAM}-\mathrm{D}_{\text {mood }}^{\mathrm{b}}$ & $2.35(3.2)$ & $0.84(2.2)$ & 0.054 & $0.55[-0.12-1.22]$ & $2.82(3.8)$ & $1.74(2.5)$ & 0.156 & $0.33[-0.33-0.99]$ \\
\hline${ }^{2} \mathrm{HAM}-\mathrm{D}_{\text {somatic }}^{\mathrm{b}}$ & $0.59(1.0)$ & $0.21(1.4)$ & 0.182 & $0.31[-0.35-0.97]$ & $0.71(1.4)$ & $0.58(1.4)$ & 0.390 & $0.09[-0.56-0.75]$ \\
\hline
\end{tabular}

Abbreviations: HTB hyperthermic baths, HAM-D Hamilton Scale for Depression

The intention-to-treat analysis used last-observation-carried-forward

${ }^{\mathrm{b}}$ Indicates differences between HTB and placebo in the change from T0 to T1/T2 to each patient's own end point for the change in depression rating

'Computed as the difference between the means, M2 - M1, divided by the pooled standard deviation, sigma ( $\left.\sigma_{\text {pooled }}\right)$ of both groups. [95\% confidence interval]

Data are shown as mean and standard deviation (SD)

\$1-tailed $t$ test

${ }^{1}$ Primary outcome

${ }^{2}$ Secondary outcome

${ }^{*} P<.05$ 
$D_{\text {total score }}$ after 4 HTB treatments compared with a sham condition. The threshold for clinical significance, as established by the National Institute for Clinical Excellence (NICE), was reached with a treatment-placebo difference of 3 points on the HAM-D [33]. Cohen $d$ was .62 (95\% CI, -0.05 to 1.29). This is larger than the effect size of antidepressant medication in a patient-level meta-analysis with $d=.37$ [34].

In clinical trials with antidepressants, an effect size of 0.40 or higher is considered a clinically significant response criterion [35]. The effect appears even stronger if we take into account that the effect sizes in published trials of antidepressant medication are $32 \%$ higher than in unpublished trials [36]. As in pharmacological studies, the magnitude of the difference in HAM-D scores between the HTB and the placebo group increases with increasing baseline depression severity (HAM-D $>22, d=1.10 ; 95 \% \mathrm{CI}, 0.02$ to 2.19 ; HAM-D $>26, d=1.67$; $95 \%$ CI, -0.07 to 3.40 ) [34]. Surprisingly, compared with the sham intervention, this did not result in a better global judgement of efficacy.

Nevertheless, these results should be interpreted with caution. First, this was a pilot study with a small sample size. Second, an improvement in HAM- $\mathrm{D}_{\text {total score }}$ does not necessarily indicate antidepressant action [37, 38]. On the other hand, our symptom-specific subscales show a statistically and clinically significant improvement in the dimensions "mood" and "insomnia", at least in the PP analysis. It is a well-known fact that the HAM$D_{\text {total score }}$ has it pitfalls, however, for better comparability with other studies, we did not use the GRID-HAM-D e.g., with better reliability and validity [39].

Regarding feasibility we did not achieve the calculated number of 44 participants in the foreseen recruiting period, the application of the hyperthermic baths was well tolerated, we saw some minor but no severe $\mathrm{AE}$, dropout rate was $18 \%$ in the HTB group and $16 \%$ in the placebo group.

The mechanism of action of HTB treatment is still unknown, but major hypotheses of WBH involve resynchronization of circadian rhythms and/or restoration of temperature physiology, resulting e.g. in better sleep [15, $16,18]$. Hence, our results are compatible with the theoretical model. We assume the difference in HAM-D scores was mainly due to an improvement in sleep quality (HAM-D $\mathrm{D}_{\text {insomnia }}$ ). This is in accordance with studies on sleep disorders in non-depressed patients [19-25].

In our study, we could not see a significant effect of HTB on HRV. This may have been due to the small sample size and the high variability of HRV parameters [40]. Different effects of HTB on HRV according to age [41] may also have influenced our results, however, the sample size was too small to perform subgroup analyses.

These findings suggest that HTB treatment improves sleep quality, mediated through a $2.4{ }^{\circ} \mathrm{C}$ increase in core body temperature. Minor and medium adverse effects were encountered these mainly being discomfort and orthostatic problems arising during or directly after the baths but also disturbed sleep in some patients the night after the intervention. Besides these transient effects, HTB treatment was well tolerated.

Our results are in line with a previously published controlled study. After 2 weeks, a single session of WBH using a radiant system, with a maximum core body temperature of $38.9^{\circ} \mathrm{C}$, resulted in a decrease in HAM$\mathrm{D}$ of 6 points, compared to a sham intervention [42]. This might indicate that different hyperthermia applications, fewer hyperthermic sessions, as well as lower core body or slightly higher skin temperatures give better results.

\section{Strengths and limitations}

The strengths of our study are the randomized, placebocontrolled design, the use of standardized baths, the good control of temperature, the clinically relevant increase in core body temperature, duration of hyperthermia of about $60 \mathrm{~min}$ and the relatively low number of dropouts.

Several limitations should be discussed. First, because of the small sample size, the study had limited power to detect clinically significant differences between the treatment conditions, especially in subgroup analyses. Second, the absence of blinding of treatment conditions, which is inherent and inevitable, and of outcome measures (only data management and statistical analyses were performed blinded). Third, the monitoring of depressive symptoms was limited to $\mathrm{T} 1$ and $\mathrm{T} 2$.

\section{Generalizability}

Although external validity may be restricted due to the population selected to participate in clinical studies, the population studied here can be regarded as representative of routine clinical practice, including patients with and without antidepressant medication [43]. Contraindications to HTB are still not well defined. Severe concomitant diseases, esp. cardiovascular, should be omitted especially in the elderly.

There is no hint that HTB treatments are less effective in combination with other pharmacological or nonpharmacological treatments, because we did not see a difference in outcome according to subgroups with or without antidepressant medication. There is also no theoretical reason that would compromise a combination with other non-pharmacological treatments.

\section{Conclusions}

In conclusion, this pilot study demonstrates effects of HTB on depressive symptoms and sleep quality in depressed patients, especially in severely depressed patients, with fast onset of treatment success after 4 treatments in 2 weeks, without severe $\mathrm{AE}$ and with 
relatively good acceptance and tolerability. With HTB treatment, the core body temperature can be raised rapidly and with clinically relevant effect $\left(2.4^{\circ} \mathrm{C}\right.$ in $\left.20 \mathrm{~min}\right)$.

Further evaluation in rigorously designed clinical studies will be necessary to validate the impact of HTB treatment on depressive disorders. Studies should preferably explore mediators and moderators of response.

\section{Additional files}

Additional file 1: Study protocol. (PDF $40 \mathrm{~kb}$ )

Additional file 2: PP analysis. Table S1. 17-Item Hamilton Scale for Depression: Differences between Baseline and T1 (after 4 Interventions) in the Hyperthermic Bath Group compared to the Placebo Group, $N=30$ ). Table S2. 17-Item Hamilton Scale for Depression: Differences between Baseline and T2 (4 weeks after treatment) in the Hyperthermic Bath Group compared to the Placebo Group, $N=28$ ). Table S3. Summary of Adverse Events. Figure S1. Effects of Hyperthermic Baths (HTB) and Green Light (Placebo) in Depressed Patients. Figure S2. Effects of Hyperthermic Baths (HTB) and Green Light (Placebo) in Depressed Patients. 17-Hamilton Scale for Depression Subscales. (DOCX 114 kb)

\section{Abbreviations}

CVD: Cardiovascular disease; HAM-D: 17-item Hamilton Scale for Depression; HRV: Heart rate variability; HTB: Hyperthermic baths; ITT: Intention-to-treat; PP: Per-protocol; SD: Standard deviation; WBH: Whole body hyperthermia

\section{Acknowledgements}

The authors are grateful to all the participants of the study. The article processing charge was funded by the German Research Foundation (DFG) and the Albert Ludwigs University Freiburg in the funding program Open Access Publishing.

\section{Availability of data and materials}

All the data supporting our findings are contained within the manuscript

\section{Authors' contributions}

$\mathrm{JN}$ : conception and design of the study; manuscript drafting, analysis and interpretation. CS: manuscript drafting, statistical analysis, interpretation, editing and final revision. JG: data acquisition, analysis and interpretation. SK: data acquisition, analysis and interpretation. TW: randomization process, statistical analysis and interpretation. $\mathrm{RH}$ : conception and design of the study, critical review of the manuscript. All authors read and approved the final manuscript.

\section{Competing interests}

JN receives support from balneology organisations such as Deutscher Heilbäderverband and Heilbäderverband Baden-Württemberg, and is a member of these organisations. None of the organisations financed this work. The authors declare that they have no competing interests.

\section{Consent for publication}

Not applicable

\section{Ethics approval and consent to participate}

Ethical approval was obtained from the local Ethics Committee (EthicsCommission Medical Center University of Freiburg; 96/13; 22.04.2013). The study was retrospectively registered in the German Clinical Trials Register (DRKS) with the registration number DRKS00004803. The study was conducted in accordance with the Declaration of Helsinki and local laws and regulations. All participants gave written informed consent. The full study protocol can be found in Additional file 1.

\section{Publisher's Note}

Springer Nature remains neutral with regard to jurisdictional claims in published maps and institutional affiliations.

\section{Author details}

'Interdisciplinary Center for Treatment and Research in Balneology, Institute for Environmental Health Sciences and Hospital Infection Control, Medical Faculty, Medical Center University of Freiburg, Breisacher Straße 115b, Freiburg im Breisgau 79106, Germany. ${ }^{2}$ Center for Complementary Medicine, Institute for Environmental Health Sciences and Hospital Infection Control, Medical Faculty, Medical Center University of Freiburg, Breisacher Straße 115b, Freiburg im Breisgau 79106, Germany.

Received: 10 September 2016 Accepted: 10 March 2017 Published online: 28 March 2017

\section{References}

1. World Health Organization (WHO) | Depression [Internet]. [cited 2016 Jun 2]. Available from: http://www.who.int/mediacentre/factsheets/fs369/en/.

2. Kessler RC, Heeringa S, Lakoma MD, Petukhova M, Rupp AE, Schoenbaum $M$, et al. The individual-level and societal-level effects of mental disorders on earnings in the United States: Results from the National Comorbidity Survey Replication. Am J Psychiatry. 2008;165:703-11.

3. National Center for Health Statistics, Preventi. Health, United States, 2013, with Special Feature on Prescription Drugs. Hyattsville: U.S. Government Printing Office; 2014.

4. Colton CW, Manderscheid RW. Congruencies in Increased Mortality Rates, Years of Potential Life Lost, and Causes of Death Among Public Mental Health Clients in Eight States. Prev. Chronic. Dis. [Internet]. 2006 [cited 2015 Dec 16];3. Available from: http://www.ncbi.nlm.nih.gov/pmc/articles/ PMC1563985/.

5. Lett HS, Blumenthal JA, Babyak MA, Sherwood A, Strauman T, Robins C, et al. Depression as a risk factor for coronary artery disease: evidence, mechanisms, and treatment. Psychosom Med. 2004;66:305-15.

6. Rudisch B, Nemeroff CB. Epidemiology of comorbid coronary artery disease and depression. Biol Psychiatry. 2003;54:227-40.

7. Harvey AG. Sleep and Circadian Functioning: Critical Mechanisms in the Mood Disorders? Annu Rev Clin Psychol. 2011;7:297-319.

8. O'Regan C, Kenny RA, Cronin H, Finucane C, Kearney PM. Antidepressants strongly influence the relationship between depression and heart rate variability: findings from The Irish Longitudinal Study on Ageing (TILDA). Psychol Med. 2015;45:623-36.

9. Van der Kooy K, van Hout H, Marwijk H, Marten H, Stehouwer C, Beekman A. Depression and the risk for cardiovascular diseases: systematic review and meta analysis. Int J Geriatr Psychiatry. 2007;22:613-26.

10. Paunio T, Korhonen T, Hublin C, Partinen M, Koskenvuo K, Koskenvuo M, et al. Poor sleep predicts symptoms of depression and disability retirement due to depression. J Affect Disord. 2015;172:381-9.

11. Souetre E, Salvati E, Wehr TA, Sack DA, Krebs B, Darcourt G. Twenty-fourhour profiles of body temperature and plasma TSH in bipolar patients during depression and during remission and in normal control subjects. Am J Psychiatry. 1988;145:1133-7.

12. Avery $\mathrm{DH}$, Wildschiødtz $\mathrm{G}$, Rafaelsen OJ. Nocturnal temperature in affective disorder. J Affect Disord. 1982:4:61-71.

13. Rush AJ, Trivedi MH, Wisniewski SR, Nierenberg AA, Stewart JW, Warden D, et al. Acute and longer-term outcomes in depressed outpatients requiring one or several treatment steps: a STAR*D report. Am J Psychiatry. 2006;163: 1905-17.

14. Hale MW, Raison CL, Lowry CA. Integrative physiology of depression and antidepressant drug action: Implications for serotonergic mechanisms of action and novel therapeutic strategies for treatment of depression. Pharmacol Ther. 2013;137:108-18.

15. Gödl R. Veränderungen der autonomen Regulation durch Überwärmungsbadtherapie bei Patienten mit depressiven Störungen. (Unpublished doctoral dissertation, University of Graz, Austria). Medizinischen Fakultät der Karl-Franzens-Universität Graz; 2000

16. Bunney JN, Potkin SG. Circadian abnormalities, molecular clock genes and chronobiological treatments in depression. Br Med Bull. 2008;86:23-32.

17. Hamilton M. Development of a Rating Scale for Primary Depressive IIIness. Br J Soc Clin Psychol. 1967;6:278-96.

18. Schaper LC. Wiederholte Hyperthermiebehandlung durch Überwärmungsbäder bei Patienten mit depressiven Störungen: Effekte auf die Produktion von Interleukin-6 sowie auf die mittlere Körpertemperatur und den psychopathologischen Befund (Doctoral dissertation, University of Freiburg, Germany). Hochschulverlag; 1996. 
19. Bunnell DE, Agnew JA, Horvath SM, Jopson L, Wills M. Passive body heating and sleep: influence of proximity to sleep. Sleep. 1988;11:210-9.

20. Horne JA, Reid AJ. Night-time sleep EEG changes following body heating in a warm bath. Electroencephalogr. Clin Neurophysiol. 1985;60:154-7.

21. Horne JA, Shackell BS. Slow wave sleep elevations after body heating: proximity to sleep and effects of aspirin. Sleep. 1987;10:383-92.

22. Sung E-J, Tochihara Y. Effects of Bathing and Hot Footbath on Sleep in Winter. J Physiol Anthropol Appl Human Sci. 2000;19:21-7.

23. Dorsey CM, Lukas SE, Teicher MH, Harper D, Winkelman JW, Cunningham $\mathrm{SL}$, et al. Effects of passive body heating on the sleep of older female insomniacs. J Geriatr Psychiatry Neurol. 1996;9:83-90.

24. Dorsey CM, Teicher MH, Cohen-Zion M, Stefanovic L, Satlin A, Tartarini W, et al. Core body temperature and sleep of older female insomniacs before and after passive body heating. Sleep. 1999;22:891-8.

25. Mishima Y, Hozumi S, Shimizu T, Hishikawa Y, Mishima K. Passive body heating ameliorates sleep disturbances in patients with vascular dementia without circadian phase-shifting. Assoc Geriatr Psychiatry. 2005;13:369-76.

26. Orme JG, Reis J, Herz EJ. Factorial and discriminant validity of the Center for Epidemiological Studies Depression (CES-D) scale. J Clin Psychol. 1986;42:28-33.

27. Hanusch K-U, Janssen CH, Billheimer D, Jenkins I, Spurgeon E, Lowry CA, et al. Whole-Body Hyperthermia for the Treatment of Major Depression: Associations With Thermoregulatory Cooling. Am J Psychiatry. 2013;170:802-4

28. Bühring M. Klinik der Hyperthermie: Untersuchungen im Überwärmungsbad. Hippokrates-Verlag; 1984.

29. American Psychiatric Association. Practice Guideline for the Treatment of Patients with Major Depressive Disorder (3rd ed.). American Psychiatric Association, Arlington; 2010.

30. Maier W, Philipp M, Gerken A. Dimensionen der Hamilton-Depressionsskala (HAMD). Eur Arch Psychiatry Neurol Sci. 1985;234:417-22.

31. Londborg PD, Smith WT, Glaudin V, Painter JR. Short-term cotherapy with clonazepam and fluoxetine: anxiety, sleep disturbance and core symptoms of depression. J Affect Disord. 2000;61:73-9.

32. Cohen J. A power primer. Psychol Bull. 1992;112:155-9.

33. National Collaborating Centre for Mental Health. Depression: management of depression in primary and secondary care. Clinical Guideline 23. National Institute for Health and Clinical Excellence. [Internet]. 2004 [cited 2016 Mar 31]. Available from: https://www.nice.org.uk/guidance/cg23.

34. Fournier JC, DeRubeis RJ, Hollon SD, Dimidjian S, Amsterdam JD, Shelton RC, et al. Antidepressant Drug effects and Depression Severity: A PatientLevel Meta-Analysis. JAMA. 2010;303:47-53.

35. Faries D, Herrera J, Rayamajhi J, DeBrota D, Demitrack M, Potter WZ. The responsiveness of the Hamilton Depression Rating Scale. J Psychiatr Res. 2000;34:3-10.

36. Turner EH, Matthews AM, Linardatos E, Tell RA, Rosenthal R. Selective Publication of Antidepressant Trials and Its Influence on Apparent Efficacy. N Engl J Med. 2008;358:252-60.

37. Prien RF, Levine J. Research and methodological issues for evaluating the therapeutic effectiveness of antidepressant drugs. Psychopharmacol Bull. 1984;20:250-7.

38. Bech P. Rating scales in depression: limitations and pitfalls. Dialogues Clin Neurosci. 2006:8:207-15.

39. Williams JBW, Kobak KA, Bech P, Engelhardt N, Evans K, Lipsitz J, et al. The GRID-HAMD: standardization of the Hamilton Depression Rating Scale. Int Clin Psychopharmacol. 2008;23:120-9.

40. Malik M, Bigger JT, Camm AJ, Kleiger RE, Malliani A, Moss AJ, et al. Heart rate variability. Eur Heart J. 1996;17:354-81

41. Nagasawa $Y$, Komori $S$, Sato M, Tsuboi $Y$, Umetani $K$, Watanabe $Y$, et al. Effects of Hot Bath Immersion on Autonomic Activity and Hemodynamics. Jpn Circ J. 2001:65:587-92.

42. Janssen CW, Lowry CA, Mehl MR, Allen JJB, Kelly KL, Gartner DE, et al. Whole-Body Hyperthermia for the Treatment of Major Depressive Disorder: A Randomized Clinical Trial. JAMA Psychiatry [Internet]. 2016 [cited 2016 May 31]; Available from: http://archpsyc.jamanetwork.com/article.aspx?doi= 10.1001/jamapsychiatry.2016.1031

43. McCambridge J, Witton J, Elbourne DR. Systematic review of the Hawthorne effect: new concepts are needed to study research participation effects. J Clin Epidemiol. 2014;67:267-77.

\section{Submit your next manuscript to BioMed Central and we will help you at every step:}

- We accept pre-submission inquiries

- Our selector tool helps you to find the most relevant journal

- We provide round the clock customer support

- Convenient online submission

- Thorough peer review

- Inclusion in PubMed and all major indexing services

- Maximum visibility for your research

Submit your manuscript at www.biomedcentral.com/submit
) Biomed Central 